\title{
On Birkhoff's Theorem in General Relativity
}

\author{
A. Barnes \\ School of Mathematics, Merz Court, The University, Newcastle upon Tyne, U.K.
}

Received March 15, 1973

\begin{abstract}
Two generalisations of Birkhoff's theorem are proved for the cases where the three-parameter group of motions acts on two-dimensional time-like and null orbits. A complete list of possible extensions of the three-parameter group to one of four parameters and of the resulting metrics is given.
\end{abstract}

\section{§ 1. Introduction}

In a series of papers Plebanski, Stachel, and Goenner [1-3] have considered space-times admitting three-parameter isometry groups with two-dimensional surfaces of transitivity. Following Goenner [3] we will denote these groups by $G_{3}(2 s), G_{3}(2 t)$ or $G_{3}(2 n)$ when the orbits are spacelike, time-like or null respectively. This class of space-times includes the spherically symmetrical case for which the well-known theorem of Birkhoff [4] is valid: a spherically symmetrical vacuum space-time admits a fourth hypersurface orthogonal Killing vector. In this paper the following generalisation of the above theorem will be proved ${ }^{1}$ : a spacetime admitting a three-parameter group of isometries with two dimensional non-null orbits and with a Ricci tensor of Segré type [(11)(11)] or [(11 11)] admits a fourth hypersurface orthogonal Killing vector. This theorem was proved in [3] for the case of space-like orbits but the analysis for time-like orbits was incorrect. An account of the algebraic classification of the Ricci tensor is given in references [5] and [6]. A vacuum space-time admitting a $G_{3}(2 n)$ is shown to be a plane-wave space-time. In the above the fourth Killing vector commutes with the original three Killing vectors (i.e. the extension of the group $G_{3}(2)$ is central). It is interesting to investigate the case where the extension is non-central. In $\S 3$ a complete list of extensions of a $G_{3}(2)$ to a $G_{4}$ and of the resulting metrics is given. This list includes the results for $G_{3}(2 n)$ and a number of other cases omitted in [3].

* Turner and Newall Research Fellow.

1 In fact for one special case mentioned at the end of $\S 2$ (ii) the theorem is not valid. 


\section{§ 2. Space-Times Admitting a $G_{3}(2)$}

(i) Space-like orbits.

For these space-times the metric may be written as [2]

$d s^{2}=-e^{2 \alpha\left(x^{3}, x^{4}\right)}\left\{\left(d x^{1}\right)^{2}+f^{2}\left(x^{1}\right)\left(d x^{2}\right)^{2}\right\}+2 \beta\left(x^{3}, x^{4}\right) d x^{3} d x^{4}$,

where $f(x)=\sin x, 1, \sinh x$, for $K=+1,0,-1$ respectively, $K$ being the Gaussian curvature of the metric $\left(d x^{1}\right)^{2}+f^{2}\left(x^{1}\right)\left(d x^{2}\right)^{2}$. The isometry group is of Bianchi type IX, VIII or VII $_{q=0}$ for $K=+1,-1$ or 0 respectively [7]. The Killing vectors are given by:

$$
\begin{aligned}
& X_{1}=\sin x^{2} \frac{\partial}{\partial x^{1}}+\cos x^{2} \cot x^{1} \frac{\partial}{\partial x^{2}}, \quad X_{2}=\frac{\partial}{\partial x^{2}}, \\
& X_{3}=\cos x^{2} \frac{\partial}{\partial x^{1}}-\sin x^{2} \cot x^{1} \frac{\partial}{\partial x^{2}}, \quad \text { for } \quad K=1, \\
& X_{1}=\frac{\partial}{\partial x^{1}}, \quad X_{2}=\frac{\partial}{\partial x^{2}}, \quad X_{3}=-x^{2} \frac{\partial}{\partial x^{1}}+x^{1} \frac{\partial}{\partial x^{2}}, \text { for } K=0 \\
& X_{1}=\sin x^{2} \frac{\partial}{\partial x^{1}}+\cos x^{2} \operatorname{coth} x^{1} \frac{\partial}{\partial x^{2}}, \quad X_{2}=\frac{\partial}{\partial x^{2}}, \\
& X_{3}=\cos x^{2} \frac{\partial}{\partial x^{1}}-\sin x^{2} \operatorname{coth} x^{1} \frac{\partial}{\partial x^{2}}, \text { for } \quad K=-1 .
\end{aligned}
$$

(ii) Time-like orbits

In this case the metric may be written as [2]

$$
\begin{aligned}
d s^{2}= & e^{2 \gamma\left(x^{3}, x^{4}\right)}\left\{f^{2}\left(x^{2}\right)\left(d x^{1}\right)^{2}-\left(d x^{2}\right)^{2}\right\} \\
& -e^{2 \beta\left(x^{3}, x^{4}\right)}\left(d x^{3}\right)^{2}-e^{2 \alpha\left(x^{3}, x^{4}\right)}\left(d x^{4}\right)^{2},
\end{aligned}
$$

where $f(x)$ is as in (2.1). The Killing vectors are given by

$$
\begin{aligned}
& X_{1}=\sinh x^{1} \frac{\partial}{\partial x^{2}}-\cosh x^{1} \cot x^{2} \frac{\partial}{\partial x^{1}}, \quad X_{2}=\frac{\partial}{\partial x^{1}}, \\
& X_{3}=\cosh x^{1} \frac{\partial}{\partial x^{2}}-\sinh x^{1} \cot x^{2} \frac{\partial}{\partial x^{1}}, \quad \text { for } \quad K=+1, \\
& X_{1}=\sinh x^{1} \frac{\partial}{\partial x^{2}}-\cosh x^{1} \operatorname{coth} x^{2} \frac{\partial}{\partial x^{1}}, \quad X_{2}=\frac{\partial}{\partial x^{1}} \\
& X_{3}=\cosh x^{1} \frac{\partial}{\partial x^{1}}-\sinh x^{1} \operatorname{coth} x^{2} \frac{\partial}{\partial x^{1}}, \text { for } \quad K=-1 .
\end{aligned}
$$


The group is of Bianchi type VIII for $K \neq 0$ and $\mathrm{VI}_{q=-1}$ for $K=0$. For $K=0$ it is convenient to use null co-ordinates in which case the metric and Killing vectors are given by

and

$$
d s^{2}=2 \gamma\left(x^{3}, x^{4}\right) d x^{1} d x^{2}-e^{2 \beta\left(x^{3}, x^{4}\right)}\left(d x^{3}\right)^{2}-e^{2 \alpha\left(x^{3}, x^{4}\right)}\left(d x^{4}\right)^{2},
$$

$$
X_{1}=\frac{\partial}{\partial x^{1}}, \quad X_{2}=\frac{\partial}{\partial x^{2}}, \quad X_{3}=-x^{1} \frac{\partial}{\partial x^{1}}+x^{2} \frac{\partial}{\partial x^{2}} .
$$

In [2] a second form of the metric is given with the first bracket in (2.3) replaced by $\left(d x^{1}\right)^{2}-f^{2}\left(x^{1}\right)\left(d x^{2}\right)^{2}$, but this metric is in fact equivalent to the first. For example the co-ordinate transformations:

$\tanh \frac{x^{2}}{2} \cosh x^{1}=\tan \frac{\tilde{x}^{1}}{2} \sinh \tilde{x}^{2}, \quad \tanh \frac{x^{2}}{2} \sinh x^{1}=\tan \frac{\tilde{x}^{1}}{2} \cosh \tilde{x}^{2} ;$

transforms the metric $\sinh ^{2} x^{2}\left(d x^{1}\right)^{2}-\left(d x^{2}\right)^{2}$ into $\left(d \tilde{x}^{1}\right)^{2}-\sin ^{2} \tilde{x}^{1}\left(d \tilde{x}^{2}\right)^{2}$. The other transformation is similar and is obtained most easily by an intermediate transformation to stereographic co-ordinates.

If $\gamma$ is not constant, curvature co-ordinates may be used so that $e^{\gamma}=x^{3}$ in Eq. (2.3) and the Einstein tensor is

$$
\begin{gathered}
G_{1}^{1}=G_{2}^{2}=-\left(\ddot{\beta}-\dot{\alpha} \dot{\beta}+\dot{\beta}^{2}\right) e^{-2 \alpha}+\left(\frac{\beta^{\prime}-\alpha^{\prime}}{x^{3}}+\alpha^{\prime} \beta^{\prime}-\alpha^{\prime 2}-\alpha^{\prime \prime}\right) e^{-2 \beta}, \\
G_{3}^{3}=-\left(\frac{2 \alpha^{\prime}}{x^{3}}+\frac{1}{\left(x^{3}\right)^{2}}\right) e^{-2 \beta}+\frac{K}{\left(x^{3}\right)^{2}}, \\
G_{4}^{4}=\left(\frac{2 \beta^{\prime}}{x^{3}}-\frac{1}{\left(x^{3}\right)^{2}}\right) e^{-2 \beta}+\frac{K}{\left(x^{3}\right)^{2}}, \\
e^{2 \alpha} G_{3}^{4}=e^{2 \beta} G_{4}^{3}=-\frac{2 \dot{\beta}}{x^{3}} e^{\beta+\alpha}
\end{gathered}
$$

where a prime and dot denote partial derivatives with respect to $x^{3}$ and $x^{4}$ respectively.

Clearly two of the eigenvalues of the Einstein tensor are equal and the condition for the other two to be equal is $\left(G_{3}^{3}-G_{4}^{4}\right)^{2}+4 G_{3}^{4} G_{4}^{3}=0$, i.e. $G_{3}^{3}=G_{4}^{4}$ and $G_{4}^{3}=G_{3}^{4}=0$. From (2.7-10) it follows that $\beta=\beta\left(x^{3}\right)$ and $\alpha^{\prime}+\beta^{\prime}=0$. Integrating we obtain $e^{\alpha}=e^{-\beta}$ where the arbitrary function of integration has been removed by a co-ordinate transformation. It is easily seen that $\frac{\partial}{\partial x^{4}}$ is a hypersurface orthogonal Killing vector and consequently the result stated in $\S 1$ is proved. The remaining field equations may be integrated easily and the results are given in Table 1.

If $\gamma$ is constant, curvature co-ordinates may not be used. However it is easily seen that the Ricci tensor necessarily is of type [(11)(11)] 
Table 1

\begin{tabular}{lllll}
\hline Ricci tensor & $e^{2 \alpha(x)}$ & & \\
\hline & $(0,0)$ & $(0, C)$ & $(R, 0)$ & $(R, C)$ \\
{$[(11)(11)]$} & - & $K-\frac{2 m}{x}+\frac{e}{x^{2}}$ & $K+a x+b x^{2}$ & $F(x)$ \\
{$[(1111)]$} & 1 & $K-\frac{2 m}{x}$ & $K-\frac{1}{3} \Lambda x^{2}$ & $K-\frac{2 m}{x}-\frac{1}{3} \Lambda x^{2}$
\end{tabular}

$(R, C)$ means that both the Ricci scalar and Weyl tensor are non-zero and a zero in either place means that the corresponding quantity vanishes.

or $[(1111)]$. For type $[(11)(11)]$ with $G_{1}^{1}$ non-constant, the metric need not admit a fourth Killing vector. The metric is the direct product of two two-dimensional metrics, one of constant curvature and the other arbitrary. For type [(11 11)] or if $G_{1}^{1}$ is constant the metric in fact admits a six parameter isometry group and is the direct product of two metrics of constant curvature. Similar results hold for the case of space-like orbits if $\alpha$ is constant in Eq. (2.1) [8].

(iii) Null orbits.

The only admissible group is Bianchi type II and the metric may be written in the form [7]:

$$
\begin{aligned}
d s^{2}= & \alpha^{2}\left(x^{3}, x^{4}\right)\left\{-2 d x^{1} d x^{4}+\left(d x^{2}\right)^{2}\right\}+\beta^{2}\left(x^{3} x^{4}\right)\left(d x^{3}\right)^{2} \\
& +2 \gamma\left(x^{3} x^{4}\right)\left(d x^{4}\right)^{2}
\end{aligned}
$$

and the Killing vectors are $X_{1}=\frac{\partial}{\partial x^{1}}, X_{2}=\frac{\partial}{\partial x^{2}}, X_{3}=x^{2} \frac{\partial}{\partial x^{1}}+x^{4} \frac{\partial}{\partial x^{2}}$. The Ricci tensor is given by

$$
\begin{gathered}
R_{1}^{1}=R_{2}^{2}=R_{4}^{4}=\frac{1}{\alpha \beta^{2}}\left\{\frac{\alpha^{\prime} \beta^{\prime}}{\beta}-\alpha^{\prime \prime}-\frac{2 \alpha^{\prime 2}}{\alpha}\right\}, \\
R_{3}^{3}=\frac{3}{\alpha \beta^{2}}\left\{\frac{\alpha^{\prime} \beta^{\prime}}{\beta}-\alpha^{\prime \prime}\right\}, \\
-\alpha^{2} R_{3}^{1}=\beta^{2} R_{4}^{3}=\frac{2}{\alpha}\left\{\frac{\alpha^{\prime} \dot{\alpha}}{\alpha}+\frac{\alpha^{\prime} \dot{\beta}}{\beta}-\dot{\alpha}^{\prime}\right\}, \\
R_{4}^{1}=\frac{1}{\alpha^{2}}\left\{\frac{\ddot{\beta}}{\beta}+\frac{\ddot{\alpha}}{\alpha}-\frac{2 \dot{\alpha}^{2}}{\alpha^{2}}-\frac{2 \dot{\alpha} \dot{\beta}}{\alpha \beta}\right\} \\
+\frac{1}{\alpha^{2} \beta^{2}}\left\{\gamma^{\prime \prime}-\frac{\beta^{\prime} \gamma^{\prime}}{\beta}-\frac{\alpha^{\prime} \gamma^{\prime}}{\alpha}+\frac{2 \gamma \alpha^{\prime} \beta^{\prime}}{\alpha \beta}-\frac{2 \gamma \alpha^{\prime \prime}}{\alpha}\right\}
\end{gathered}
$$


where a prime and dot denote partial derivatives with respect to $x^{3}$ and $x^{4}$ respectively. The Weyl tensor is type $N$ or zero as there is an isotropy group acting on null orbits [9]. The Ricci tensor has three equal eigenvalues and is of one of the following Segré types [(31)], [(21) 1], [(111) 1] or their degeneracies. If $R_{a b}=A l_{a} l_{b}$ where $l^{a}=\delta_{1}^{a}$ it follows from (2.12)-(2.14) that $\alpha^{\prime}=0$ and this implies that the null vector $l^{a}$ is constant. We make use of this result to prove the following theorem: a vacuum space-time admitting a $G_{3}(2 n)$ is a plane-wave space-time.

Proof. The co-ordinate transformations which preserve the form of the Killing vectors are $\tilde{x}^{1}=x^{1}+f\left(x^{3}, x^{4}\right) ; \tilde{x}^{2}=x^{2} ; \tilde{x}^{3}=\psi\left(x^{3}, x^{4}\right): \tilde{x}^{4}=x^{4}$; and the metric transforms as follows:

$$
\begin{gathered}
\tilde{\alpha}=\alpha, \quad \beta=\tilde{\beta} \psi^{\prime}, \quad-\tilde{\alpha}^{2} f^{\prime}+\tilde{\beta}^{2} \psi^{\prime} \dot{\psi}+\tilde{g}_{34} \psi^{\prime}=0, \\
2 \gamma=2 \tilde{\gamma}+\tilde{\beta}^{2} \dot{\psi}^{2}+2 \tilde{g}_{34} \dot{\psi}-2 \tilde{\alpha}^{2} \dot{f} .
\end{gathered}
$$

By a suitable choice of $f$ and $\psi$ we may put $\tilde{g}_{34}=0$ and $\tilde{\beta}=\tilde{\beta}\left(x^{4}\right)$ and the remaining co-ordinate freedom is

$$
\tilde{x}^{1}=x^{1}+\frac{\beta^{2}}{\alpha^{2}}\left\{\frac{1}{2} \frac{\dot{A}}{A}\left(x^{3}\right)^{2}+\frac{\dot{B}}{A} x^{3}+C\right\}, \quad \tilde{x}^{3}=A x^{3}+B,
$$

where $A, B$ and $C$ are arbitrary functions of $x^{4}$ only.

The field Eq. (2.15) implies that

$2 \gamma=-\beta^{2}\left\{\frac{\ddot{\alpha}}{\alpha}-\frac{2 \dot{\alpha}^{2}}{\alpha^{2}}+\frac{\ddot{\beta}}{\beta}-\frac{2 \dot{\alpha} \dot{\beta}}{\alpha \beta}\right\}\left(x^{3}\right)^{2}+F\left(x^{4}\right) x^{3}+G\left(x^{4}\right)$,

where $F$ and $G$ are arbitrary functions of integration. From (2.16) (2.17) it follows that $\tilde{\gamma}$ may be set equal to zero if the functions $A, B$ and $C$ are chosen to satisfy the equations

$$
\begin{array}{r}
\frac{\ddot{A}}{A}-\frac{2 \dot{A}^{2}}{A^{2}}+2\left(\frac{\dot{\beta}}{\beta}-\frac{\dot{\alpha}}{\alpha}\right) \frac{\dot{A}}{A}+\frac{\ddot{\alpha}}{\alpha}-\frac{2 \dot{\alpha}^{2}}{\alpha^{2}}+\frac{\ddot{\beta}}{\beta}-\frac{2 \dot{\alpha} \dot{\beta}}{\alpha \beta}=0, \\
\frac{\ddot{B}}{A}-\frac{2 \dot{B} \dot{A}}{A^{2}}+2\left(\frac{\dot{\beta}}{\beta}-\frac{\dot{\alpha}}{\alpha}\right) \frac{\dot{B}}{A}+\frac{F}{2 \beta^{2}}=0, \\
2 \dot{C}+4\left(\frac{\dot{\beta}}{\beta}-\frac{\dot{\alpha}}{\alpha}\right) C-\frac{\dot{B}^{2}}{A}+\frac{G}{\beta^{2}}=0 .
\end{array}
$$

Thus the metric is of type $\mathrm{VI}_{2}$ in Table 3 and consequently admits two additional Killing vectors, one of which $\left(\frac{\partial}{\partial x^{3}}\right)$ is hypersurface orthogonal. The metric is in fact a plane wave and the transformations to the usual metric form [9] are

$$
\begin{aligned}
& u=\int \alpha^{2}\left(x^{4}\right) d x^{4}, \quad v=x^{1}+\frac{1}{2} \frac{\dot{\alpha}}{\alpha^{3}}\left(x^{2}\right)^{2}+\frac{1}{2} \frac{\dot{\beta}}{\alpha^{2} \beta}\left(x^{3}\right)^{2} \\
& x=\alpha x^{2}, \quad y=\beta x^{3} .
\end{aligned}
$$




\begin{tabular}{|c|c|c|c|}
\hline$G_{3}$ & $G_{4}$ & Commutation relations & Killing vector \\
\hline IX & VIII & {$\left[X_{a} Y\right]=0$} & $-\frac{\partial}{\partial x^{3}}+\varepsilon \frac{\partial}{\partial x^{4}} \quad \varepsilon=0$ or 1 \\
\hline VIII & VII & {$\left[X_{a} Y\right]=0$} & $\begin{array}{cccc}-\frac{\partial}{\partial x^{3}}+\varepsilon \frac{\partial}{\partial x^{4}} & \text { for } & G_{3}(2 s) \\
\frac{\partial}{\partial x^{4}} & \text { for } & G_{3}(2 t)\end{array}$ \\
\hline \multirow[t]{2}{*}{$\mathrm{VII}_{q=0}$} & $\mathrm{VI}_{4}(k=\varepsilon=0)$ & {$\left[X_{a} Y\right]=0$} & $-\frac{\partial}{\partial x^{3}}+\varepsilon \frac{\partial}{\partial x^{4}} \quad \varepsilon=0$ or 1 \\
\hline & $\mathrm{V}$ & {$\left[X_{1} Y\right]=X_{1}\left[X_{2} Y\right]=X_{2}\left[X_{3} Y\right]=0$} & $x^{1} \frac{\partial}{\partial x^{1}}+x^{2} \frac{\partial}{\partial x^{2}}-\frac{\partial}{\partial x^{3}}+\varepsilon \frac{\partial}{\partial x^{4}}$ \\
\hline \multirow[t]{2}{*}{$\mathrm{VI}_{q}=-1$} & $\mathrm{VI}_{1,5}(c=-1 \quad e=0)$ & {$\left[X_{a} Y\right]=0$} & $\frac{\partial}{\partial x^{4}}$ \\
\hline & IV & {$\left[X_{1} Y\right]=0\left[X_{2} Y\right]=X_{2}\left[X_{3} Y\right]=0$} & $x^{2} \frac{\partial}{\partial x^{2}}+\frac{\partial}{\partial x^{4}}$ \\
\hline \multirow[t]{4}{*}{ II } & I $(c \neq 2)$ & {$\left[X_{1} Y\right]=c X_{1}\left[X_{2} Y\right]=X_{2}\left[X_{3} Y\right]=(c-1) X_{3}$} & $c x^{1} \frac{\partial}{\partial x^{1}}+x^{2} \frac{\partial}{\partial x^{2}}+(2-c) x^{4} \frac{\partial}{\partial x^{4}}$ \\
\hline & II & {$\left[X_{1} Y\right]=2 X_{1}\left[X_{2} Y\right]=X_{2}\left[X_{3} Y\right]=X_{2}+X_{3}$} & $2 x^{1} \frac{\partial}{\partial x^{1}}+x^{2} \frac{\partial}{\partial x^{2}}-\frac{\partial}{\partial x^{4}}$ \\
\hline & III & {$\left[X_{1} Y\right]=q X_{1}\left[X_{2} Y\right]=X_{3}\left[X_{3} Y\right]=-X_{2}+q X_{3}$} & $\left(q x^{1}+\frac{1}{2}\left(x^{2}\right)^{2}\right) \frac{\partial}{\partial x^{1}}+\left(q x^{2}-x^{2} x^{4}\right) \frac{\partial}{\partial x^{2}}+\left(q x^{4}-1+\left(x^{4}\right)^{2}\right) \frac{\partial}{\partial x^{4}}$ \\
\hline & $\mathrm{VI}_{3}(k=0)$ & {$\left[X_{1} Y\right]=0\left[X_{2} Y\right]=0\left[X_{3} Y\right]=X_{2}$} & $-\frac{\partial}{\partial x^{4}}$ \\
\hline \multirow[t]{3}{*}{ II } & $\mathrm{I}(c=2)$ & {$\left[X_{1} Y\right]=2 X_{1}\left[X_{2} Y\right]=X_{2}\left[X_{3} Y\right]=X_{3}$} & $2 x^{1} \frac{\partial}{\partial x^{3}}+\frac{\partial}{\partial x^{3}}$ \\
\hline & $\mathrm{VI}_{2}(k=\varepsilon=0)$ & $\begin{array}{l}{\left[X_{a} Y\right]=0} \\
\text { also admits fifth Killing vector } Z\end{array}$ & $Y=\frac{\partial}{\partial x^{3}}$ \\
\hline & & {$\left[X_{1} Z\right]=\left[X_{2} Z\right]=\left[X_{3} Z\right]=0 \quad[Y, Z]=X_{1}$} & $Z=x^{3} \frac{\partial}{\partial x^{1}}+f\left(x^{4}\right) \frac{\partial}{\partial x^{3}}$ with $f_{4}=\alpha^{2} / \beta^{2}$ in (2.11) \\
\hline
\end{tabular}


Table 3

\begin{tabular}{|c|c|c|c|c|c|}
\hline$G_{4}$ & Metric & & & & Orbit \\
\hline VIII, VII, VI $_{4} \varepsilon=1$ & $\alpha=\alpha\left(x^{3}+x^{4}\right)$ & $\beta=\beta\left(x^{3}+x^{4}\right)$ & & in $(2.1)$ & $T$ or $S$ \\
\hline VIII, VII, VI $_{4} \varepsilon=0$ & $\alpha=1$ & $\beta=\beta\left(x^{4}\right)$ & & in $(2.1)$ & $N$ \\
\hline VII & $\alpha=\alpha\left(x^{3}\right)$ & $\beta=\beta\left(x^{3}\right)$ & $\gamma=\gamma\left(x^{3}\right)$ & in $(2.3)$ & $T$ \\
\hline $\mathrm{VI}_{1,5}$ & $\alpha=\alpha\left(x^{3}\right)$ & $\beta=\beta\left(x^{3}\right)$ & $\gamma=\gamma\left(x^{3}\right)$ & in $(2.5)$ & $T$ \\
\hline IV & $\alpha=\alpha\left(x^{3}\right)$ & $\beta=\beta\left(x^{3}\right)$ & $\gamma=\tilde{\gamma}\left(x^{3}\right) e^{2 x^{4}}$ & in $(2.5)$ & $T$ \\
\hline $\mathrm{V} \varepsilon=1$ & $\alpha=\tilde{\alpha}\left(x^{3}+x^{4}\right) x^{3}$ & $\beta=\beta\left(x^{3}+x^{4}\right)$ & & in $(2.1)$ & $T$ or $S$ \\
\hline $\mathrm{V} \varepsilon=0$ & $\alpha=\tilde{\alpha}\left(x^{4}\right) x^{3}$ & $\beta=\beta\left(x^{4}\right)$ & & in $(2.1)$ & $N$ \\
\hline I $c \neq 2$ & $\alpha=\tilde{\alpha}\left(x^{3}\right)\left(x^{4}\right)^{-\frac{2}{2-c}}$ & $\beta=1$ & $\gamma=\tilde{\gamma}\left(x^{3}\right) /\left(x^{4}\right)^{2}$ & in $(2.11)$ & $T$ \\
\hline II & $\alpha=\tilde{\alpha}\left(x^{3}\right) e^{x^{4}}$ & $\beta=1$ & $\gamma=\gamma\left(x^{3}\right)$ & in $(2.11)$ & $T$ \\
\hline \multirow[t]{2}{*}{ III } & $\alpha=\tilde{\alpha}\left(x^{3}\right)\left(1-q x^{4}+\right.$ & $\left.+\left(x^{4}\right)^{2}\right)^{-\frac{1}{2}} e^{-q p}$ ta & $a^{-1} p\left(2 x^{4}-q\right)$ & $\beta=1$ & \\
\hline & $\gamma=\tilde{\gamma}\left(x^{3}\right)\left\{1-q x^{4}\right.$ & $\left.+\left(x^{4}\right)^{2}\right\}^{-2}$ where & $p=\left(4-q^{2}\right)^{-\frac{1}{2}}$ & in $(2.11)$ & $T$ \\
\hline $\mathrm{VI}_{3}$ & $\alpha=\alpha\left(x^{3}\right)$ & $\beta=1$ & $\gamma=\gamma\left(x^{3}\right)$ & in $(2.11)$ & $T$ \\
\hline I $(c=2)$ & $\alpha=\tilde{\alpha}\left(x^{4}\right) e^{-x^{3}}$ & $\beta=\beta\left(x^{4}\right)$ & $\gamma=0$ & in $(2.11)$ & $N$ \\
\hline $\mathrm{VI}_{2}$ & $\alpha=\alpha\left(x^{4}\right)$ & $\beta=\beta\left(x^{4}\right)$ & $\gamma=0$ & in $(2.11)$ & $N$ \\
\hline
\end{tabular}

In the last column a $T, S$ or $N$ denotes that the three-dimensional orbit of the $G_{4}$ is time-like, space-like or null respectively. For a $G_{4}$ containing a $G_{3}(2 s)$ the orbit is spacelike, time-like or null when the component of the additional Killing vector orthogonal to the two-dimensional orbit is space-like, time-like or null respectively.

\section{§ 3. Extensions of a $G_{3}(2)$ to a $G_{4}$}

Let $X_{a} a=1,2,3$ and $Y$ be the original and additional Killing vectors respectively. Then

$$
\left[X_{a} Y\right]=b_{a}^{b} X_{b}+d_{a} Y
$$

where $b_{b}^{a}$ and $d_{a}$ are constants. By using the Jacobi identities and the freedom of choice of $Y$ (it may be replaced by an arbitrary linear combination of the $X_{a}$ 's and $Y$ ) it is possible to classify all extensions of the original Lie algebras. Many of the extensions are not compatible with the structure equations and Killing's equations and only the admissible extensions together with the form of the additional Killing vector are given in Table 2. In Table 3 the resulting metric forms for all extensions are given. It is an immediate consequence of the generalised Birkhoff's theorem that if an extension of type IV or V (see Ref. [7]) is complete then the Ricci tensor is not of Segré type [(11) (11)].

For those extensions of a group of Bianchi type II which have timelike three-dimensional orbits the Ricci tensor is not of type [(31)] but the other two types [(21) 1], [(111) 1] and their degeneracies can all occur. For those extensions with null orbits the Ricci tensor has four equal eigenvalues. More specifically for type $\mathrm{VI}_{2}$ the Ricci tensor can be written as $R^{a b}=A l^{a} l^{b}$ where $l^{a}\left(=\delta_{1}^{a}\right)$ is a null vector. For type $I_{c=2}$ all 
A. Barnes

three Segré types [(31)], [(211)] and [(1111)] occur but in this case the eigenvalue is non-zero.

We note that for all central extensions the additional Killing vector is hypersurface orthogonal but that this result is not true for non-central extensions unless additional conditions are satisfied.

Acknowledgement. I would like to thank Dr. R. A. Russell-Clark for help in computing the Ricci tensors in $\S 2$.

\title{
References
}

1. Plebanski, J., Stachel, J.: J. Math. Phys. 9, 269-283 (1968)

2. Goenner, H., Stachel, J.: J. Math. Phys. 11, 3358-3370 (1970)

3. Goenner, H.: Commun. math. Phys. 16, $34-47$ (1970)

4. Birkhoff, G.D.: Relativity and modern physics, pp. 253-256. Cambridge Univ. Press 1927

5. Plebanski, J.: Acta. Phys. Polon. 26, 963-1020 (1964)

6. Barnes, A.: General Relativity and Gravitation. 5, (1974) to appear.

7. Petrov, A. Z.: Einstein spaces. London: Pergamon 1969

8. Foyster, J. M., McIntosh, C. B. G.: Commun. math. Phys. 27, 241-246 (1972)

9. Ehlers, J., Kundt, W.: Chapter 2. In: Witten, L. (Ed.): Gravitation, An introduction to current research. New York: Wiley 1962

\author{
A. Barnes \\ School of Mathematics \\ Merz Court \\ Newcastle upon Tyne, NE 1, 7 RU, UK
}

